\title{
Upper limits on the diffuse gamma-rays measured with KASCADE-Grande
}

D. Kang ${ }^{* 1}$, W.D. Apel ${ }^{1}$, J.C. Arteaga-Velázquez ${ }^{2}$, K. Bekk ${ }^{1}$, M. Bertaina ${ }^{3}$, J. Blümer ${ }^{1,4}$, H. Bozdog ${ }^{1}$, I.M. Brancus ${ }^{5}$, E. Cantoni ${ }^{3,6}$, A. Chiavassa ${ }^{3}$, F. Cossavella ${ }^{4}$, K. Daumiller ${ }^{1}$, V. de Souza ${ }^{7}$, F. Di Pierro ${ }^{3}$, P. Doll ${ }^{1}$, R. Engel ${ }^{1}$, Z. Feng ${ }^{8}$, D. Fuhrmann ${ }^{9}$, A. Gherghel-Lascu ${ }^{5}$, H.J. Gils ${ }^{1}$, R. Glasstetter ${ }^{9}$, C. Grupen ${ }^{10}$, A. Haungs ${ }^{1}$, D. Heck ${ }^{1}$, J.R. Hörandel ${ }^{11}$, D. Huber ${ }^{4}$, T. Huege ${ }^{1}$, K.-H. Kampert ${ }^{9}$, H.O. Klages ${ }^{1}$, K. Link ${ }^{4}$, P. Łuczak ${ }^{12}$, H.J. Mathes ${ }^{1}$, H.J. Mayer ${ }^{1}$, J. Milke ${ }^{1}$, B. Mitrica ${ }^{5}$, C. Morello ${ }^{6}$, J. Oehlschläger ${ }^{1}$, S. Ostapchenko ${ }^{13}$, N. Palmieri' ${ }^{4}$, T. Pierog ${ }^{1}$, H. Rebel $^{1}$, M. Roth ${ }^{1}$, H. Schieler ${ }^{1}$, S. Schoo ${ }^{1}$, F.G. Schröder ${ }^{1}$, O. Sima ${ }^{14}$, G. Toma ${ }^{5}$, G.C. Trinchero ${ }^{6}$, H. Ulrich ${ }^{1}$, A. Weindl ${ }^{1}$, J. Wochele ${ }^{1}$, J. Zabierowski ${ }^{12}$ KASCADE-Grande Collaboration

${ }^{1}$ Institut für Kernphysik, KIT - Karlsruhe Institute of Technology, Germany

${ }^{2}$ Universidad Michoacana, Inst. Física y Matemáticas, Morelia, Mexico

${ }^{3}$ Dipartimento di Fisica, Università degli Studi di Torino, Italy

${ }^{4}$ Institut für Experimentelle Kernphysik, KIT - Karlsruhe Institute of Technology, Germany

${ }^{5}$ Horia Hulubei National Institute of Physics and Nuclear Engineering, Bucharest, Romania

${ }^{6}$ Osservatorio Astrofisico di Torino, INAF Torino, Italy

${ }^{7}$ Universidade São Paulo, Instituto de Física de São Carlos, Brasil

${ }^{8}$ Institute of High Energy Physics, Beijing, China

${ }^{9}$ Fachbereich Physik, Universität Wuppertal, Germany

${ }^{10}$ Department of Physics, Siegen University, Germany

${ }^{1}$ Dept. of Astrophysics, Radboud University Nijmegen, The Netherlands

12 National Centre for Nuclear Research, Department of Astrophysics, Lodz, Poland

${ }^{13}$ Frankfurt Institute for Advanced Studies (FIAS), Frankfurt am Main, Germany

${ }^{14}$ Department of Physics, University of Bucharest, Bucharest, Romania

E-mail: donghwa.kang@kit.edu

KASCADE-Grande was a multi-detector array to measure individual air showers of cosmic rays in the energy range of $10 \mathrm{PeV}$ up to $1 \mathrm{EeV}$. Based on full data sets measured by KASCADEGrande, an upper limit to the flux of ultra-high energy gamma-rays in the primary cosmic rays is determined. The analysis is performed by selecting air showers with low muon contents due to a small fraction of secondary hadrons in gamma ray showers to cosmic ray showers. A preliminary result on the $90 \%$ C.L. upper limit to the relative intensity of gamma-rays with respect to cosmicray primaries will be presented and discussed with limits reported in previous measurements in this contribution.

The 34th International Cosmic Ray Conference,

30 July- 6 August, 2015

The Hague, The Netherlands

* Speaker. 


\section{Introduction}

Investigations of the galactic and extragalactic diffuse emission are potentially able to give information about the source and propagation of galactic cosmic rays. The measured flux of diffuse gamma-rays and its spectrum thus may provide new insights into the acceleration of cosmic rays. Direct measurements of ultra-high energy photons by satellite or balloon-borne experiments are difficult due to the low fluxes. This detection can, however, be accomplished by large area groundbased detectors using the air shower array technique.

For the diffuse flux of ultra-high energy gamma radiation, there are two main plausible sources: First source is due to decays of neutral pions produced by the collision of cosmic-rays with interstellar gas and dust in the disk of the Galaxy. In this case, the predicted integral intensity is concentrated in the galactic plane. The second one is due to electromagnetic cascades resulting from the interactions of extremely high-energy cosmic-rays with the $2.7 \mathrm{~K}$ cosmological microwave background radiation or topological defects in early Universe. In this case, this results in an isotropic flux of secondary photons uniformly distributed over cosmological distances. Therefore, the measurements of the diffuse isotropic gamma-ray flux might provide information on the ultra-high energy cosmic-ray components. In addition, this flux would represent a background for experiments searching for the gamma-ray enhancement from the direction of the galactic disk.

Extensive air showers are mainly characterized by the total electron number and the total muon number. In general, muons are produced by the decay of charged kaons or pions, which in hadronic showers are produced in nucleus-nucleus interactions, whereas in photon showers only in photoproduction processes. The ratio between the cross sections of photoproduction and nucleusnucleus interaction processes is very small, in the order of $\sim 10^{-3}$. Therefore, the usual strategy for searches for primary gamma-rays in extensive air showers is to discriminate gamma-ray primaries from the hadronic background by identifying muon-poor extensive air showers.

This contribution presents upper limits on the relative intensity of the gamma-ray component of cosmic rays measured by the KASCADE-Grande experiment. In the analysis, nearly five times more data sets of the gamma-ray simulations are used, compared to the one for the result in Ref. [1], whereas the same method of the analysis is applied.

\section{The KASCADE-Grande experiment}

KASCADE-Grande was an extensive air shower experiment located at Karlsruhe Institute of Technology ( $49.1^{\circ}$ north, $8.4^{\circ}$ east, $110 \mathrm{~m}$ above sea level). It operated until the end of 2012 and all components were by now dismantled.

The KASCADE-Grande [2] array covering an area of $700 \times 700 \mathrm{~m}^{2}$ was optimized to measure extensive air showers up to primary energies of $1 \mathrm{EeV}$. It comprised 37 scintillation detector stations located on a hexagonal grid with an average spacing of $137 \mathrm{~m}$ for the measurements of the electromagnetic and muonic shower components. Each of the detector stations was equipped with plastic scintillators covering a total area of $10 \mathrm{~m}^{2}$. The angular resolution of KASCADE-Grande is better than $0.5^{\circ}$ over the whole energy range, so that it will allow us to perform also gamma-ray searches. 


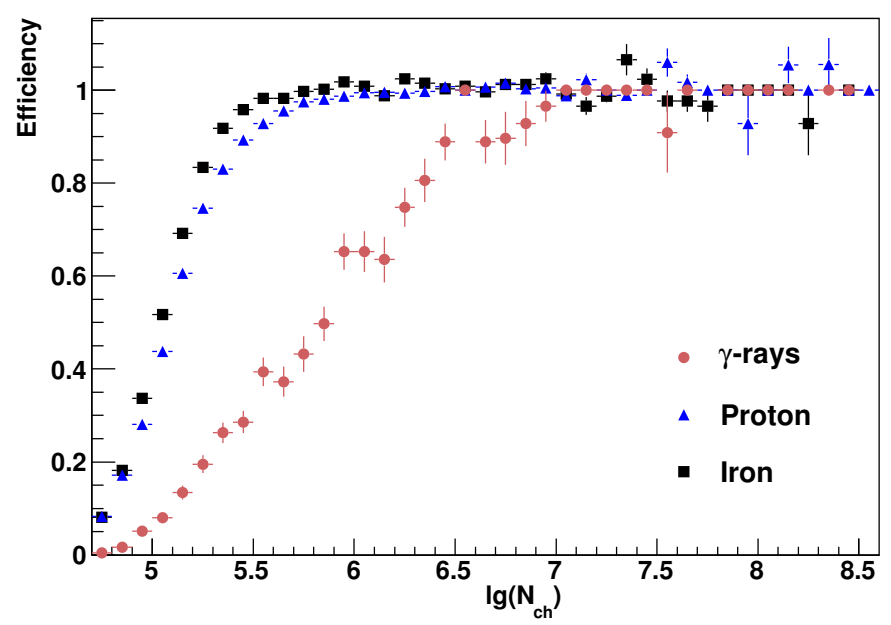

Figure 1: Trigger and reconstruction efficiency as a function of the number of charged particles for air showers induced by photons, protons and iron primaries.

\section{Gamma and cosmic ray simulations}

An essentail part of the present analysis is the Monte-Carlo simulation of the experiment. For the simulation of the physical processes in the air shower development the CORSIKA [3] program has been used, applying hadronic interaction models. To determine the signals in the individual detectors, all secondary particles at the ground level are passed through a complete detector simulation program using the GEANT package. The predicted observables at ground level, such as e.g. the number of electrons, muons and photons are then compared to the measurements.

The FLUKA [4] $(\mathrm{E}<200 \mathrm{GeV})$ model has been used for hadronic interactions at low energies. High-energy interactions were treated with different models QGSJETII-2 [5]. Showers initiated by primary photons, protons, $\mathrm{He}, \mathrm{CNO}, \mathrm{Si}$ and iron nuclei have been simulated. The simulations covered the energy range of $10^{14}-10^{18} \mathrm{eV}$ with zenith angles in the interval $0^{\circ}-60^{\circ}$. The spectral index in the simulations was -2 and for the analysis it is weighted to a slope of -3 . The same number of showers was generated for each particle type.

\subsection{Efficiency}

The trigger and reconstruction efficiency of KASCADE-Grande as a function of the shower size, i.e. the number of charged particles, is demonstrated in Fig. 1. The efficiency is defined by the ratio of the number of events in the reconstructed area to the number of events in the true area, in which the binomial statistical errors are used. Efficiencies above one are due to cuts on the reconstructed core positions, i.e. events falling outside the selected area can be reconstructed inside. Full efficiency is reached at the number of charged particles of around $10^{6}$ for air showers induced by protons and iron primary particles, which approximately corresponds to a primary energy of $10^{16} \mathrm{eV}$. However, for showers induced by photon primaries the full efficiency is higher due to the 
missing muon trigger at large distances. The limit at high energies is due to the restricted area of the Grande array.

\section{The analysis and result}

\subsection{Selection of data}

For this analysis full data sets taken from 2003 to 2012 were used, where only successfully reconstructed and precisely measured events were selected. The core positions of the showers are inside a circular area of $152,214 \mathrm{~m}^{2}$ around the center of the array to avoid large reconstruction errors at the edges of the detector field. The zenith angle is smaller than $40^{\circ}$ to ensure full efficiency. After applying all quality cuts, we obtained in total ca. $1.7 \times 10^{7}$ events for a measurement time of ca. 1865 days.

\subsection{Gamma hadron discrimination}

A scattered diagram of the observed number of muons $\lg \left(N_{\mu}\right)$ and charged particles $\lg \left(N_{c h}\right)$ is shown in Fig. 2. The simulated gamma-ray showers are superimposed as well. The blue data points indicate the whole experimental data set, whereas the red one illustrate the simulated gamma-ray events. The shower size is corrected to a zenith angle of $20^{\circ}$ using the method of constant intensity [6].

Since gamma-ray induced air showers are notable for their lack of muons, compared to hadronic showers, we select a data sample enriched in photon showers by rejecting showers containing muons, i.e., we therefore take a more conservative way in which the expected background is not subtracted from the event number below the cut line.

The selection of muon-poor showers is indicated by the straight line in Fig. 2. This selection is motivated by the simulated gamma-rays and its optimization is still under investigation. The events below the straight line were taken into consideration for further analysis. They amount to 1060 out of a total of 17 millions events. In the region below this line the events are expected to be mainly due to primary photons because air showers induced by heavy nuclei show a larger muon to electron ratio.

\subsection{Upper limit of $I_{\gamma} / I_{C R}$}

No possible excess of events consistent with a gamma-ray signal was seen in the data. Hence, we assume that all events below the selection line are background and set upper limits on the gamma-ray fraction of the cosmic rays. We estimate the $90 \%$ C.L. upper limit on the number of observed events, applying standard statistical methods [7] and the efficiency for gamma-ray detection.

Assuming that all events passing the gamma-ray selections are indeed gamma-rays, the $90 \%$ upper limit on the fraction of the gamma-ray integral flux relative to the cosmic-ray integral flux, $I_{\gamma} / I_{C R}$, is estimated by [7]

$$
\frac{I_{\gamma}}{I_{C R}}<\frac{N_{90}}{N_{t o t} \varepsilon_{\gamma}}\left(\frac{E_{C R}}{E_{\gamma}}\right)^{-\beta+1}
$$




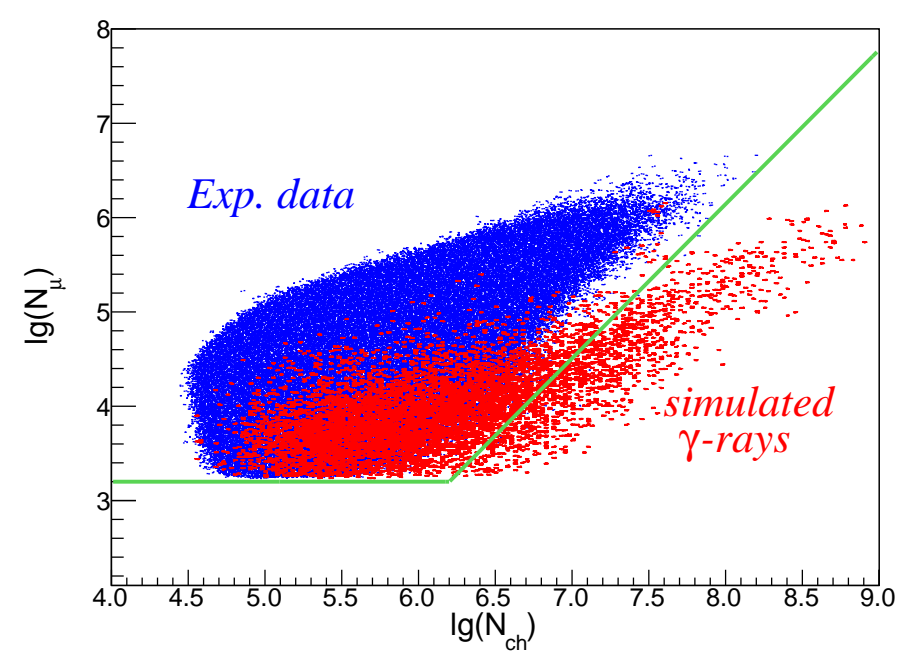

Figure 2: Scatter distribution of the measured number of muons $\lg \left(N_{\mu}\right)$ and number of charged particles $\lg \left(N_{c h}\right)$, superimposed with simulated gamma-ray showers. The green line indicates the selection criteria for the subset of the muon-poor showers.

\begin{tabular}{llccccc}
\hline \hline $\lg \left(N_{c h}\right)$ & $N_{\text {tot }}$ & $N_{90}$ & $E_{C R}$ & $E_{\gamma}$ & $I_{\gamma} / I_{C R}$ & $I_{\gamma}\left(\times 10^{-17}\right)$ \\
\hline$>6.5$ & $6.19 \times 10^{6}$ & 358 & $3.21 \times 10^{4}$ & $1.38 \times 10^{4}$ & $<1.88 \times 10^{-5}$ & $<1.19$ \\
$>7$ & 85537 & 351 & $8.72 \times 10^{4}$ & $3.29 \times 10^{4}$ & $<6.79 \times 10^{-4}$ & $<8.51$ \\
$>7.5$ & 9640 & 214 & $2.21 \times 10^{5}$ & $8.30 \times 10^{4}$ & $<2.08 \times 10^{-3}$ & $<8.64$ \\
$>8$ & 1239 & 122 & $5.31 \times 10^{5}$ & $1.98 \times 10^{5}$ & $<1.03 \times 10^{-2}$ & $<8.32$ \\
$>8.5$ & 165 & 78 & $1.13 \times 10^{5}$ & $2.92 \times 10^{5}$ & $<2.48 \times 10^{-2}$ & $<3.89$ \\
\hline \hline
\end{tabular}

Table 1: Results of the search for diffuse ultra-high energy gamma-rays at different threshold values of $N_{c h}$. The mean cosmic-ray energy, $E_{C R}$, and the mean gamma-ray energy, $E_{\gamma}$, are given in the unit of TeV. $I_{\gamma} / I_{C R}$ is the $90 \%$ C.L. upper limit on the integral gamma-ray fraction, and $I_{\gamma}$ is the $90 \%$ C.L. upper limit on the integral gamma-ray flux in the unit of photons $\mathrm{cm}^{-2} \mathrm{~s}^{-1} \mathrm{sr}^{-1}$.

where $N_{90}$ is the $90 \%$ C.L. upper limit on the number of detected events, $\varepsilon_{\gamma}$ is the efficiency for gamma-ray detection, and $\beta$ is the integral cosmic-ray spectral index $\left(\beta=3.0, E_{\gamma}>4 \mathrm{PeV}\right) . E_{C R}$ and $E_{\gamma}$ are the mean energies of cosmic-ray and gamma-ray, respectively, which produce the same shower size $\left(N_{c h}\right)$. Therefore, they are calculated for a fixed $N_{c h}$ bin using the resulting Gaussian function.

The results of the search for diffuse ultra-high energy gamma-rays for different threshold values of $N_{c h}$ are summarized in Table 1. To determine upper limits to the integral flux of gamma-rays at fixed gamma-ray energies, we use measurements of the all-particle primary energy spectrum of Ref. [8]. The flux limits are also given in Table 1.

Figure 3 displays the measurements on the gamma-ray fraction as a function of energy, including this work, for the energy range of $10 \mathrm{PeV}$ up to $300 \mathrm{PeV}$. The limits presented here represent the first results in the energy range above $5 \times 10^{16} \mathrm{eV}$. 


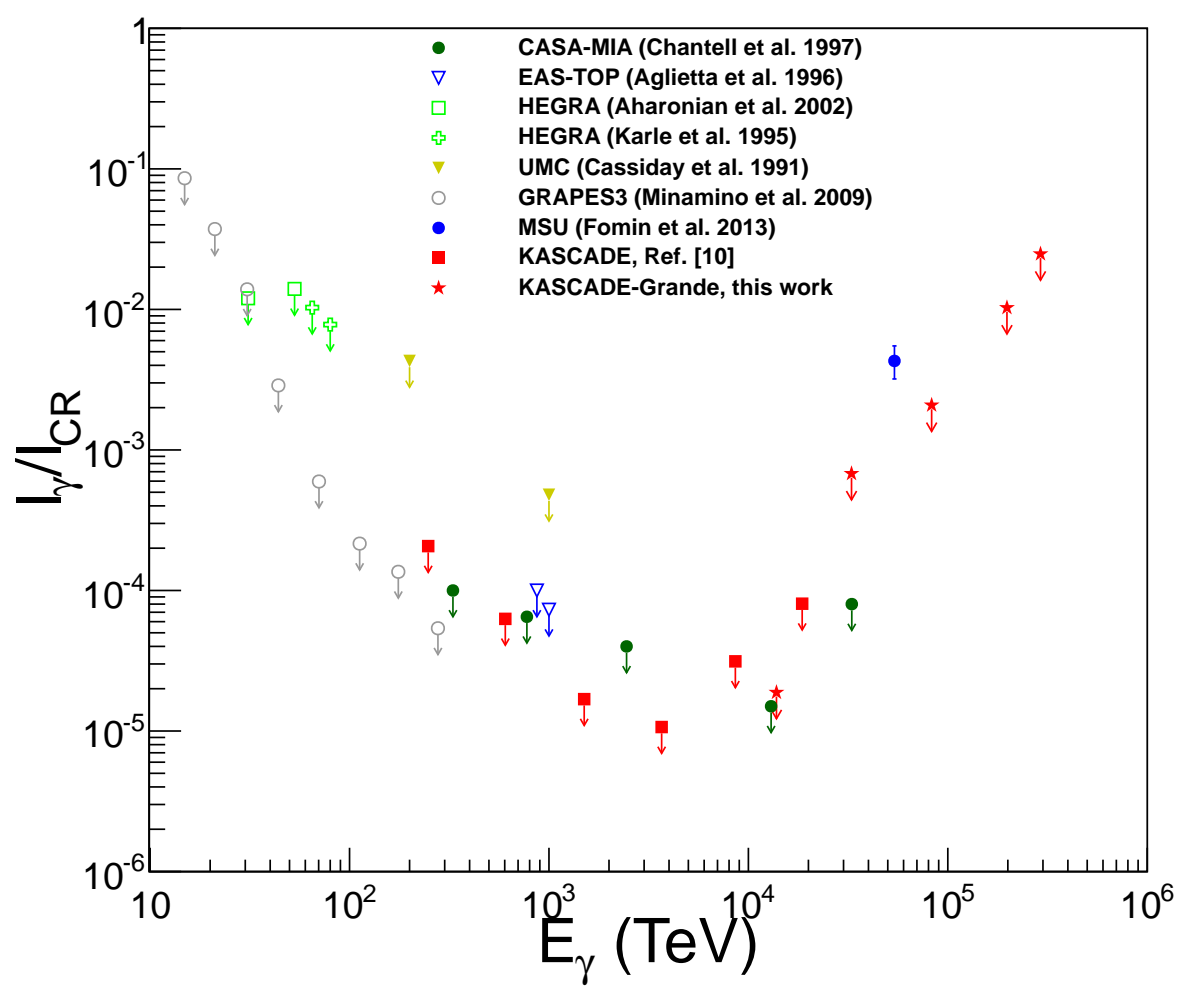

Figure 3: Fraction of gamma-rays relative to the cosmic rays. The red stars represent the results from KASCADE-Grande.

These results are compared with other previous experiments $[12,13]$ and with theoretical curves by a specific IceCube excess model [11] in Fig. 4. The lines are the IceCube excess models coming from different distances of sources of neutrinos in the galaxy, where these neutrinos are also responsible for primary gamma-rays. The updated results [10] of the KASCADE experiment with more data are compatible with the theoretical prediction of the IceCube excess model coming from $<20 \mathrm{kpc}$ in the galactic plane. It might hence set some constrains on the distance of sources for the IceCube Excess. Details will be described elsewhere in a forthcoming publication.

It should be noted that all values in Fig. 4 are upper limits, except the one from MSU [14]; This reported positive signal seems to be disproved by the presented results.

\section{Conclusion}

Using full data sets measured by the KASCADE-Grande experiment, the $90 \%$ C.L. upper limits to the diffuse flux of ultra-high energy gamma-rays for the energy range of $10 \mathrm{PeV}$ to 300 $\mathrm{PeV}$ are determined by selecting showers with low muon contents.

The best upper limit to the fraction of the gamma-ray to the cosmic-ray flux is obtained: $I_{\gamma} / I_{C R}<1.88 \times 10^{-5}$ for $13.8 \mathrm{PeV}$. These stringent limits are the first results in the energy range above $10 \mathrm{PeV}$, and might constrain a limit to the background rate of muon-poor showers in the search for the galactic disk enhancement of cosmic rays. Comparison of these experimental upper 


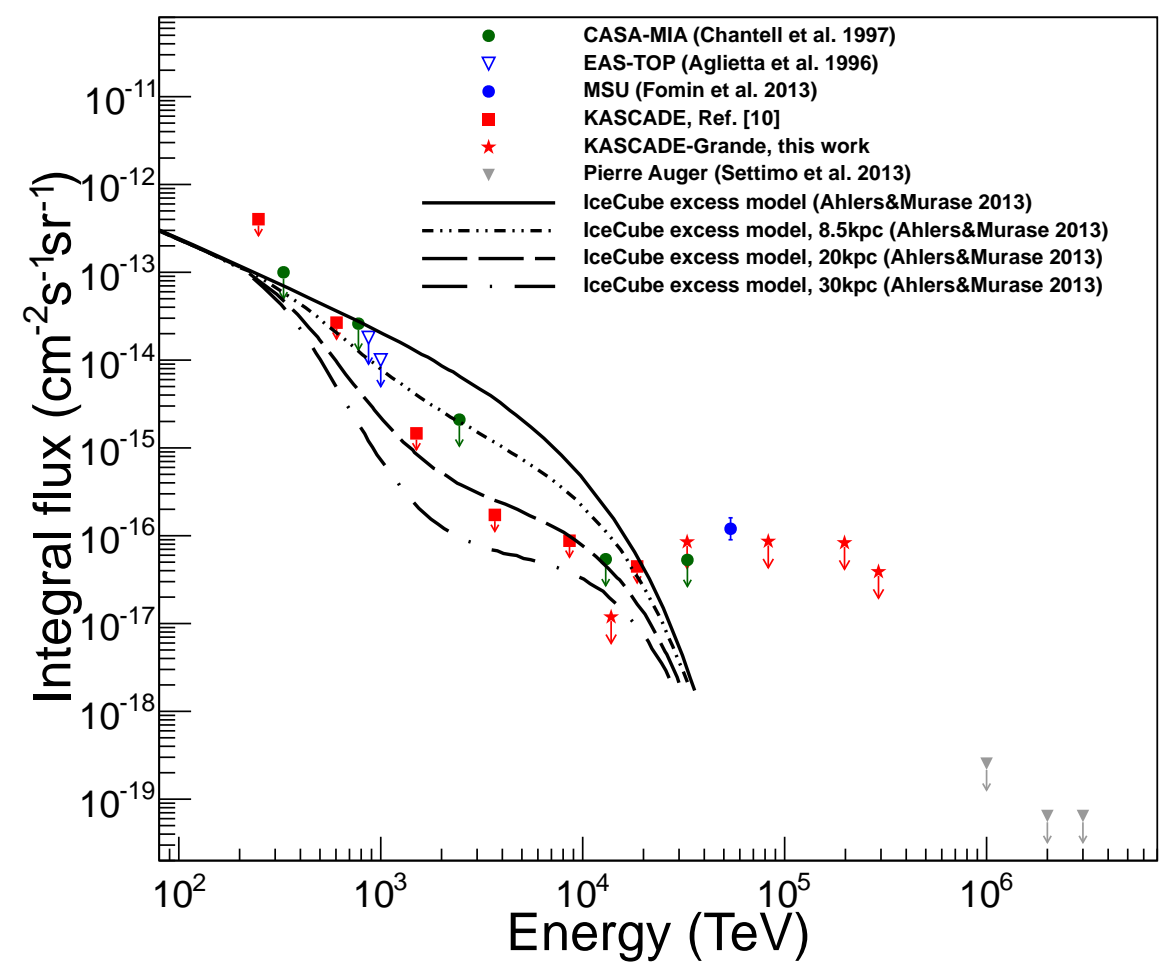

Figure 4: Comparison of integral flux of gamma-rays with previous results and with theoretical curves by an IceCube excess model [11].

limits with the theoretical predictions is still required, since no theoretical predictions exist so far at energies around $10 \mathrm{PeV}$. These preliminary results will be improved by a more careful gammahadron discrimination and also by a new muon reconstruction.

In addition, the results [10] of the original KASCADE experiment are updated by including eight years of more data since the publication in Ref. [9]. It should be mentioned that this might set some constraints on the distance of sources for the IceCube excess.

\section{Acknowledgments}

The authors would like to thank the members of the engineering and technical staff of the KASCADE-Grande collaboration, who contributed to the success of the experiment. The KASCADEGrande experiment is supported in Germany by the BMBF and by the 'Helmholtz Alliance for Astroparticle Physics - HAP' funded by the Initiative and Networking Fund of the Helmholtz Association, by the MIUR and INAF of Italy, the Polish Ministry of Science and Higher Education, and the Romanian Authority for Scientific Research UEFISCDI (PNII-IDEI grants 271/2011 and 17/2011). J.C.A.V. acknowledges the partial support of CONACyT. 


\section{References}

[1] D. Kang et al. KASCADE-Grande Collaboration, Proc. 24th European Cosmic Ray Symposium, Kiel (2014)

[2] W.D. Apel et al. KASCADE-Grande Collaboration, Nucl. Instr. Meth. A 620 (2010) 202

[3] D. Heck et al., Rep. FZKA 6019, Forschungszentrum Karlsruhe (1998)

[4] A. Fassò et al., CERN-2005-10, INFN/TC-05/11, SLAC-R-773 (2005)

[5] S.S. Ostapchenko, Phys. Rev. D 74 (2006) 014026

[6] W.D. Apel et al. KASCADE-Grande Collaboration, Astropart. Phys. 36 (2012) 183

[7] O. Helene, Nucl. Instr. Meth. 212 (1983) 319

[8] W.D. Apel et al. KASCADE-Grande Collaboration, Phys. Rev. Lett. 107171104 (2011)171104

[9] G. Schatz et al. KASCADE Collaboration, Proc. 28th Int. Cosmic Ray Conf., Tsukuba, Japan (2003)

[10] Z. Feng et al. KASCADE Collaboration, 34th Int. Cosmic Ray Conf., The Hague, The Netherlands (2015) \#0811 these proceedings

[11] M. Ahlers and K. Murase, arXiv:1309.4077v3 (2014)

[12] M. Aglietta et al., Astopart. Phys. 6 (1996) 71

[13] M.C.Chantell et al., Phys. Rev. Lett. 79 (1997) 1805

[14] Yu. A. Fomin, arXiv:1307.4988v2 (2013) 